2 BiolD analysis of the cyclin $\mathrm{F}$ interactome reveals that ALS-variant cyclin $\mathrm{F}$

$5 \quad$ Stephanie L. Rayner ${ }^{1 *}$, Flora Cheng ${ }^{1}$, Shu Yang ${ }^{1}$, Natalie Grima ${ }^{1}$, Yazi D. Ke ${ }^{3}$, Carol G.

$6 \mathrm{Au}^{3}$, Marco Morsch ${ }^{1}$, Alana De Luca ${ }^{1}$, Jennilee M. Davidson ${ }^{1}$, Mark P. Molloy ${ }^{2}$, Bingyang

$11{ }^{1}$ Department of Biomedical Sciences, Centre for Motor Neuron Disease Research,

12 Faculty of Medicine and Health Sciences, Macquarie University, 2 Technology Place,

13 North Ryde, NSW 2109

$14{ }^{2}$ Faculty of Medicine and Health, Sydney School of Medicine, Royal North Shore Hospital,

15 Pacific Hwy, St Leonards, Sydney, NSW 2065

$16{ }^{3}$ Department of Biomedical Sciences, Dementia Research Centre, Faculty of Medicine

17 and Health Sciences, Macquarie University, 2 Technology Place, North Ryde, NSW 2109

\title{
Authors and emails:
}

20 Stephanie L. Rayner: stephanie.rayner@mq.edu.au

21 Flora Cheng: flora.cheng@mq.edu.au

22 Shu Yang: shu.yang@mq.edu.au

23 Natalie Grima: natalie.grima@mq.edu.au

24 Yazi D. Ke: yazi.ke@mq.edu.au

25 Carol G. Au: carol.au@mq.edu.au 
26 Marco Morsch: marco.morsch@mq.edu.au

27 Alana De Luca: alana.deluca@utas.edu.au

28 Jennilee M. Davidson: jennilee.davidson@hdr.mq.edu.au

29 Mark P. Molloy: m.molloy@sydney.edu.au

30 Bingyang Shi: bingyang.shi@mq.edu.au

31 Lars M. Ittner: lars.ittner@mq.edu.au

32 Ian Blair: ian.blair@mq.edu.au

33 Roger S. Chung: roger.chung@mq.edu.au

34 Albert Lee: albert.lee@mq.edu.au

Highlights

- Previously, we identified missense mutations in CCNF that are linked to Amyotrophic lateral sclerosis/Frontotemporal dementia (ALS/FTD) and have shown that a single mutation in cyclin $\mathrm{F}$ can cause defects to major protein degradation systems in dividing cells.

- Mass spectrometry and bioinformatic studies demonstrate that cyclin F interacts with several RNA binding proteins. This includes the essential paraspeckle proteins, RBM14. Notably, this interaction could be validated by standard immunoprecipitations and immunoblotting. Cyclin F could also be found to interact with a series of essential proteins which form the paraspeckle complex. 
- We further evaluated the effect of cyclin $\mathrm{F}(\mathrm{S} 621 \mathrm{G})$ on the homeostasis of these novel interaction partners in primary neurons in response to a known paraspeckle inducer, MG132. Notably, we demonstrate significant defects in the homeostasis of RBM14 and SFPQ, but not NONO, when cyclin F carries an S621G mutation.

- Unlike other paraspeckle proteins, RBM14 levels have not previously been reported in the post-mortem brain and spinal cord of ALS patient post-mortem tissue. Here, we note significant defects in the homeostasis of RBM14 in the postmortem tissue of ALS patients.

\section{Abstract}

61 Background: Previously, we identified missense mutations in CCNF that are causative of

62 familial and sporadic amyotrophic lateral sclerosis (ALS) and frontotemporal dementia

63 (FTD). CCNF encodes for the protein cyclin F, a substrate recognition component of the

64 E3-ubiquitin ligase, SCF cyclin F. We have previously shown that mutations in CCNF cause

65 disruptions to overall protein homeostasis; causing a build-up of ubiquitylated proteins (1)

66 as well as defects in autophagic machinery (2).

Methods: Here, we have used an unbiased proteomic screening workflow using BiolD, as well as standard immunoprecipitations to identify novel interaction partners of cyclin F, identifying the interaction between cyclin $\mathrm{F}$ and a series of paraspeckle proteins. The

71 homeostasis of these new cyclin F interaction partners, RBM14, NONO and SFPQ were 72 monitored in primary neurons using immunoblotting. In addition, the homeostasis of

73 RBM14 was compared between control and ALS/FTD patient tissue using standard IHC 74 studies. 
76 Results: Using BiolD, we found over 100 putative interaction partners of cyclin $\mathrm{F}$ and

77 demonstrated that cyclin $\mathrm{F}$ closely associates with a number of essential paraspeckle

78 proteins, which are stress-responsive proteins that have recently been implicated in ALS

79 pathogenesis. We further demonstrate that the turnover of these novel binding partners

80 are defective when cyclin F carries an ALS/FTD-causing mutation. In addition the analysis

81 of RBM14 levels in ALS patient post-mortem tissue revealed that RBM14 levels were

82 significantly reduced in post-mortem ALS patient motor cortex and significantly reduced in

83 the neurons of spinal cord tissue.

84

85 Conclusion: Overall, our data demonstrate that the dysregulation of paraspeckle

86 components may be contributing factors to the molecular pathogenesis of ALS/FTD.

87

88 Keywords: BiolD, cyclin F, paraspeckles, RBM14, amyotrophic lateral sclerosis,

89 frontotemporal dementia, proteomics, ubiquitylation, homeostasis.

90

91 Materials and correspondence

92 Albert Lee (albert.lee@mq.edu.au)

93 Stephanie L. Rayner (stephanie.rayner@ma.edu.au)

94 Current address: 2 Technology Place, North Ryde, NSW 2109, Australia. 


\section{Background}

102 Amyotrophic lateral sclerosis (ALS) is typically a late-onset neurodegenerative disease

103 characterised by the selective degeneration of upper and lower motor neurons of the

104 cerebral cortex, brainstem and spinal cord. It is the most common form of motor neurone

105 disease (MND) with poor prognosis and limited treatment options. A proportion of ALS

106 patients also develop clinical or subclinical frontotemporal dementia (FTD) and

107 pathological and genetic overlap is now recognised, indicating that they represent a

108 spectrum of disease (3). Approximately $5-10 \%$ of ALS patients carry an autosomal

109 dominant genetic mutation. Familial mutations have been reported in over 30 genes

110 including $\operatorname{SOD1}(4,5), \operatorname{VCP}(6), \operatorname{TARDBP}(7), \operatorname{FUS}(8,9), \operatorname{OPTN}(10), \operatorname{SQSTM1}(11)$,

111 UBQLN2 (12), MATR3 (13) and TBK1 $(14,15)$. Identification of these genes has drawn

112 attention to protein clearance pathways, proteins that accumulate within insoluble

113 cytoplasmic inclusions and defects in RNA processing in disease pathogenesis. Recently,

114 we identified several novel missense mutations in CCNF in patients with ALS/FTD (1).

115 CCNF encodes for cyclin F, a 786 amino acid protein that forms part of the multi-protein

116 Skp1-Cul1-F-Box (SCF $\left.{ }^{\text {cyclin }} \mathrm{F}\right)$ E3 ligase that is known to regulate cell cycle progression

117 through timely ubiquitylation of substrates to regulate their homeostasis through

118 proteasomal degradation (16).

120 We have previously reported that a familial ALS/FTD mutation in cyclin F (denoted cyclin

$121 \mathrm{~F}^{\mathrm{S} 621 \mathrm{G}}$ ) alters the ubiquitylation activity of $\mathrm{SCF}^{\mathrm{cyclin}} \mathrm{F}$, leading to the accumulation of

122 ubiquitylated proteins (1). In addition, we have also shown that the activity of cyclin $\mathrm{F}$ may

123 be regulated by post-translational modifications and that the loss of a phosphorylation site

124 causes aberrant ubiquitylation activity (2). Ultimately this leads to defects in bulk 
125 degradation processes and an upregulation in caspase-mediated cell death pathways 126 (17).

128 Currently, there are few known interaction partners of cyclin F. These proteins are 129 generally associated with cell-cycle function, including substrates such as ribonucleoside130 diphosphate reductase subunit M2 (RRM2) (18), nucleolar and spindle-associated protein 1311 (NuSAP) (19), centriolar coiled-coil protein of $110 \mathrm{kDa}$ (CP110) (20), cell division control 132 protein 6 homolog (CDC6) (21), stem-loop binding protein (SLBP) (22), exonuclease 1 133 (exo1) (23) and fizzy-related protein homolog (Fzr1) (24). In addition, known interaction 134 partners of cyclin F include Skp1 (forming part of the ubiquitin ligase complex), b-myb (25) 135 and CKII (26). Given that the interaction partners of cyclin F that have been reported to 136 date are predominantly involved in cell-cycle regulation, it is not immediately obvious how 137 cyclin $\mathrm{F}^{\mathrm{S} 621 \mathrm{G}}$ might trigger neurodegeneration in non-dividing neurons. Therefore, we hypothesised that there are other interaction partners of cyclin $F$ that may help to understand the processes that may become defective in non-dividing cells.

141 BiolD can be used to identify protein interaction partners and proteins in close proximity 142 ( $\sim 10 \mathrm{~nm}$ radius) (27) to the protein of interest using an engineered biotin-ligase, BirA* (28,

143 29). An advantage of using BiolD over standard immunoprecipitation (IP) methods, is the

144 ability to identify transient, low abundance interaction partners as well as proteins that are

145 not soluble in standard IP buffers (30). In recent years, BiolD has been used to identify 146 novel binding partners of a number of proteins including lamin A (31) and E-cadherin (32), 147 ZO-1 (33), TDP-43 and fragmented TDP-43 (34). In addition, BiolD has been utilized to 148 identify substrates of $\beta$-TrCP 1 and 2 (35). 
150 In this study, we have used BiolD followed by mass spectrometry (MS) to characterise the

151 interactome of cyclin F. In doing so, we identified more than 100 putative interaction

152 partners of cyclin $\mathrm{F}$, including a group of RNA binding proteins that are also essential

153 paraspeckle proteins. Previously we have demonstrated that an ALS-causing mutation in

154 cyclin $F$ causes defects in major protein degradation systems, thus we evaluate the

155 dysregulation of these proteins in primary neurons and in ALS patient tissue.

METHODS

160 Plasmids and Cloning

161 Expression constructs encoding wild type and S621G CCNF cDNA fused to an N-terminal

162 mCherry fluorophore were used as described previously (1). Wild type and S621G CCNF

163 cDNA fused to a C-terminal Flag-tag was also cloned into a pcDNA 3.1 vector. BirA* alone

164 or BirA* in frame with cyclin $\mathrm{F}$ was cloned into pcDNA5/FRT/TO. Constructs encoding

165 RBM14-HA were cloned into a pcDNA3.1 vector.

167 Cell culture

168 Human Embryonic Kidney Cells (HEK293) and HEK293 Flp-In T-Rex cells were grown

169 and maintained in Dulbecco's modified Eagle medium (DMEM, Sigma-Aldrich)

170 supplemented with $10 \%(\mathrm{v} / \mathrm{v})$ of heat-inactivated fetal bovine serum (FBS, Sigma-Aldrich).

171 Plated cells were grown and maintained in a humidified incubator held at a constant

172 temperature of $37^{\circ} \mathrm{C}$, with $5 \% \mathrm{CO}_{2}$. All cell lines were tested for mycoplasma prior to

173 experimental work using the MycoAlert Mycoplasma Detection Kit (Lonza). 
175 In order to generate stably-transfected cell lines, HEK293 Flp-In T-Rex cells (Thermo)

176 were double-transfected using constructs encoding Flp-recombinase (pOG44) as well as

177 constructs encoding BirA*-cyclin F using Lipofectamine 2000 (Thermo) according to the

178 manufacturer's instructions. After 48 hours, cells were selected with $100 \mu \mathrm{g} / \mathrm{mL}$

179 Hygromycin (InvivoGen) and $15 \mu \mathrm{g} / \mathrm{mL}$ Blasticidin (InvivoGen). In order to ensure the cells

180 were stably-transfected and that transgene expression could be induced, tetracycline

181 (Sigma-Aldrich) was added to cell culture media at a final concentration of $0.1 \mu \mathrm{g} / \mathrm{mL}$ for

182 18-24 hours. Tetracycline-dependent gene expression was monitored using standard

183 immunoblotting procedures.

184

185 Primary cell culture

186 Primary mouse cortical neurons were cultured as previously described (36). Briefly, brains

187 were obtained from embryos on embryonic day 16.5. Cerebral hemispheres were sub-

188 dissected, digested in trypsin at $37^{\circ} \mathrm{C}$ and homogenized using fire-polished glass pipettes

189 into single cell suspension. Cells were seeded out at 5 million cells per $10 \mathrm{~cm}$ dish in

190 medium containing 10\% FBS/high glucose DMEM (Life Technologies). Medium was

191 changed 2 hours post seeding and cells were subsequently maintained in Neurobasal

192 medium supplemented with Glutamax and B27 supplement (Life Technologies).

193

194 Proximity-labelling in live HEK293 Flp-In T-Rex cells

195 Stably transfected HEK293 Flp-In T-Rex cells were grown and maintained in DMEM

196 supplemented with 10\% FBS and $100 \mu \mathrm{g} / \mathrm{mL}$ Hygromycin (InvivoGen) and $15 \mu \mathrm{g} / \mathrm{mL}$

197 Blasticidin (InvivoGen). Once cells reached 70\% confluency, expression of BirA* or BirA*-

198 cyclin F (wild-type and CCNF variants) was induced by adding $0.1 \mu \mathrm{g} / \mathrm{mL}$ of tetracycline

199 (Sigma-Aldrich) to cell culture media. In order to biotinylate proteins in proximity to the 
200 transgene, $50 \mu \mathrm{M}$ of biotin (Sigma-Aldrich) was simultaneously added to the culture

201 media. After 18-24 hours, cells were washed with PBS and harvested into ice-cold PBS.

202 Harvested cells were washed twice with ice-cold PBS and centrifuged at $2000 \times g$ for 10

203 minutes at $4^{\circ} \mathrm{C}$. Washed cell pellets were snap frozen at $-80^{\circ} \mathrm{C}$ until further use.

205 Total cell lysis

206 For total cell lysis, frozen cell pellets were first defrosted on ice and resuspended in ice207 cold modified RIPA buffer (50 mM Tris-HCl, 150 mM NaCl, 1\% NP-40, 1mM EDTA, 1mM

208 EGTA, 0.1\% SDS, 0.5\% Sodium deoxycholate, $\mathrm{pH} 7.4)$ containing appropriate amounts of 209 protease and phosphatase inhibitor cocktails (Roche). Cells were incubated in RIPA buffer

210 for 15 minutes on ice with intermittent vortexing before probe sonication using a Sonic

211 Ruptor 250 at $50 \%$ power and pulser settings set to $30 \%$. Lysates were subject to a total

212 of 10 pulses each before centrifugation at $14,000 \times \mathrm{g}$ for 20 minutes at $4^{\circ} \mathrm{C}$. The

213 supernatant containing cellular proteins was aliquoted and stored at $-80^{\circ} \mathrm{C}$ until further 214 analysis.

\section{Biotin pull-downs}

217 Cleared lysates containing biotinylated proteins in modified RIPA buffer were incubated 218 with $30 \mu \mathrm{L}$ of pre-washed streptavidin-coated magnetic beads (Thermo Fisher) for 3 hours

219 at $4^{\circ} \mathrm{C}$ whilst rotating. In order to isolate biotinylated proteins from the complex mixture, a 220 magnetic rack was used to isolate magnetic beads. Isolated magnetic beads were washed

2215 times in modified RIPA buffer. Captured biotinylated proteins were eluted by 222 resuspension in Laemmli sample buffer (BioRad), containing NuPAGE Sample Reducing 223 Agent (Invitrogen) and were boiled at $95^{\circ} \mathrm{C}$ for 10 minutes. The eluents were prepared for 224 1D SDS-PAGE as described below. 


\section{Immunoprecipitations}

226 HEK293 cells were transfected with constructs encoding mCherry-cyclin F, Flag-cyclin F

227 or RBM14-HA using Lipofectamine 2000 according to the manufacturer's instructions.

228 Transfected cells were harvested after 24 hours and cell pellets were resuspended in NP.

22940 lysis buffer (1 \% (v/v) Nonidet P-40 in Tris-buffered saline (TBS), 2 mM EDTA, 230 cOmplete protease inhibitor cocktail and phosSTOP (Roche)). The resuspended cells

231 were vortexed, then probe sonicated (10 seconds, Setting 3, Branson Sonifier 450). The

232 cell lysates were centrifuged at $14,000 \times g$ for 30 minutes to remove cell debris. For

233 immunoprecipitations, approximately $500 \mu \mathrm{g}$ of cellular protein was incubated with $1 \mathrm{ug}$ of

234 flag antibody or $20 \mu \mathrm{L}$ of RFP-Trap® ${ }^{\circledR}$ MA (Chromotek). The magnetic beads were

235 collected using a magnet and washed three times in NP-40 lysis buffer. For western blot

236 analysis, beads were resuspended in 1x Loading buffer (BioRad) containing 1x reducing

237 reagent (NuPage) and boiled at $95^{\circ} \mathrm{C}$ for 10 minutes.

238

239

240 SDS PAGE and Immunoblotting

241 Equal amounts of protein were separated on a $4-12 \%$ Bis-Tris SDS PAGE gel. Proteins

242 were transferred onto a nitrocellulose membrane using a Bio-Rad Trans-blot Turbo semi-

243 dry transfer cell (1.3 A, $25 \mathrm{~V}, 7$ mins). The membranes were blocked in $3 \%$ skim milk

244 powder in PBST for half an hour prior to incubation with primary antibody overnight at $4^{\circ} \mathrm{C}$

245 or 1 hour at RT. Primary antibodies used in this study were: rabbit polyclonal anti-cyclin F

246 (1:300; cat\# sc-952, Santa Cruz Biotechnology), mouse monoclonal anti-mCherry (1:300;

247 cat\# 632543, Clonetech), mouse monoclonal anti- $\beta$-actin (Abcam, dilution- 1:12,000,

248 catalogue \#ab6276-101), mouse monoclonal anti-GAPDH (Proteintech, dilution-

249 1:10,000), mouse monoclonal anti-a-tubulin (Sigma-Aldrich, dilution- 1:1000, catalogue 
250 \#T5168), rabbit polyclonal anti-RBM14 (Sigma-Aldrich, dilution- 1:1000, catalogue

251 \#HPA006628), mouse monoclonal anti-PSPC1 (Santa Cruz, dilution- 1:500, catalogue

252 \#sc-374367), mouse monoclonal anti-PSF (Santa Cruz, dilution- 1:1000, catalogue \#sc-

253 101137), rabbit polyclonal anti-Matrin 3 (Proteintech, dilution- 1:1000, catalogue \#12202-

$2542-A P)$.

255

256 After incubation with primary antibodies, the membranes were washed in PBS-T three

257 times for 10 minutes before fluorescently labelled IRDye 800CW Goat Anti-Rabbit IgG

258 Secondary Antibody $\left(1: 15,000 ;\right.$ LI-COR) or fluorescently labelled IRDye ${ }^{\circledR} 680 R D$ Goat

259 anti-Mouse IgG Secondary Antibody (1:15,00; LI-COR) secondary antibodies was added

260 for 30 minutes at RT. Immunoblots were imaged using a Li-Cor Odyssey imaging system

261 at the appropriate wavelength.

262

263 In-gel trypsin digestion

264 Equal amounts of protein were loaded and separated on a 4-15\% SDS-PAGE gel

265 (BioRad). The resulting gel was briefly incubated in fixing solution (50\% methanol, 10\%

266 acetic acid) and proteins were stained with Coomassie blue R250 until protein bands were

267 visible. The gel was then left to destain overnight in Destain solution (25\% methanol).

268 After destaining, protein bands were excised from gels into 5 fractions. Gel fractions were

269 then cut into smaller pieces $\left(\sim 1 \mathrm{~mm}^{2}\right)$ and further destained with $50 \%$ methanol/50 $\mathrm{mM}$

270 ammonium bicarbonate $(\mathrm{pH}$ 8). Gel pieces were then washed and dehydrated in $50 \%$

271 acetonitrile $(\mathrm{ACN}) / 50 \mathrm{mM}$ ammonium bicarbonate for 10 minutes, then incubated with

$272100 \%$ ACN until gel pieces were completely dehydrated. ACN was removed, and gel

273 pieces were dried under vacuum centrifugation before being incubated with $10 \mathrm{mM}$

274 dithiothreitol (DTT) in $50 \mathrm{mM}$ ammonium bicarbonate (AmBic) for 40 minutes at $37^{\circ} \mathrm{C}$. 
275 Excess DTT was removed before gel pieces were incubated with $25 \mathrm{mM}$ iodoacetamide

276 (IAA) in $50 \mathrm{mM}$ ammonium bicarbonate for 40 minutes at room temperature in the dark.

277 Gel pieces were then washed twice with 50\% ACN/50 mM ammonium bicarbonate for 10

278 minutes each time before the supernatant was removed and gel pieces were incubated in $279100 \%(v / v)$ ACN to dehydrate gel pieces as described earlier. Excess ACN was removed 280 and gel pieces were left to dry.

281

282 Gel pieces were incubated with trypsin (12.5 ng/ $\mu$; proteomics grade, Sigma-Aldrich) 283 diluted in $50 \mathrm{mM}$ ammonium bicarbonate and incubated overnight at $37^{\circ} \mathrm{C}$. After 284 incubation, the supernatant was transferred into fresh tubes and acidified with formic acid 285 (FA). The gel pieces were incubated in 50\% ACN, 2\% FA. Supernatants containing tryptic 286 peptides were pooled and lyophilised. For desalting, peptides were resuspended in $0.1 \%$ 287 FA and desalted using pre-washed and equilibrated C18 OMIX tips (Agilent). Once 288 desalted, samples were again lyophilised and stored at $-80^{\circ} \mathrm{C}$ until MS analysis.

290 Prior to mass spectrometry, lyophilised peptides were resuspended in $0.1 \%$ FA and bath 291 sonicated for 20 minutes. The resuspended peptides were then centrifuged at $14,000 \times g$ 292 for 15 minutes to remove any insoluble debris, and the clarified peptides were analysed by 293 LC-MS/MS. The peptide fractions were separated on an Ultimate 3000 nanoLC (Thermo 294 Fisher Scientific) fitted with the Acclaim PepMap RSLC column (Thermo Fisher Scientific), 295 making use of a 60 minutes gradient (2-95\% v/v acetonitrile, $0.1 \% \mathrm{v} / \mathrm{v}$ formic acid) 296 running at a flow rate of $300 \mathrm{nl} /$ minute. Peptides eluted from the nano LC column were 297 subsequently ionized into the $Q$ Exactive ${ }^{\text {TM }}$ Plus mass spectrometer (Thermo Fisher 298 Scientific). The electrospray source was fitted with an emitter tip 10 $\mu$ m (New Objective, 299 Woburn, MA) and maintained at $1.5 \mathrm{kV}$ electrospray voltage. The temperature of the 
300 capillary was set to $250^{\circ} \mathrm{C}$. Precursor ions were selected for MS/MS fragmentation using a 301 data-dependent "Top 10" method operating in FT-FT acquisition mode with HCD 302 fragmentation. FT-MS analysis on the $\mathrm{Q}$ Exactive ${ }^{\mathrm{TM}}$ Plus was carried out at 70,000 303 resolution and an AGC target of $1 \times 10^{6}$ ions in full MS. MS/MS scans were carried out at 30417,500 resolution with an AGC target of $2 \times 10^{4}$ ions. Maximum injection times were set to 30530 and 50 milliseconds respectively. The ion selection threshold for triggering MS/MS 306 fragmentation was set to 25,000 counts and an isolation width of $2.0 \mathrm{Da}$ was used to perform HCD fragmentation with normalised collision energy of 27.

Bioinformatics and statistics

310 The raw files were searched using Proteome Discoverer 2.4 software (Thermo Fisher 311 Scientific) incorporating the Sequest search algorithm employing the Homo sapiens 312 Uniprot FASTA databases. Peptide identifications were determined taking into account a 313 20-ppm precursor ion tolerance and 0.1 Da MS/MS fragment ion tolerance for FT-MS and 314 HCD fragmentation respectively. Peptide modifications were also considered whereby 315 cysteine carbamidomethylation was considered a static modification. Variable 316 modifications included methionine oxidation, asparagine and glutamine deamidation, 317 lysine biotinylation, and acetylated N-terminal residues. Trypsin was set as the enzyme of 318 use, allowing for three missed cleavages at the most. Data was also processed using a 319 label-free quantitation (LFQ) workflow employing the Minora Feature node, making use of 320 a Protein FDR validator node which estimates the false discovery rates at the protein level 321 as well as a percolator node to estimate the FDR at the PSM level. Results were adjusted 322 so that the final global FDR was less than $1 \%$ at the protein and peptide level. A $q$-value 323 of 0.01 was required to validate protein identifications. 
325 Statistical analyses were typically conducted using GraphPad Prism 8.2.1 software or

326 Ingenuity Pathway Analysis (IPA). In GraphPad Prism, statistical analyses involved the

327 use of a paired t-test. Comparisons were considered significant if the $p$-values were less

328 than 0.05 .

329

330 Statistically significant protein functions were identified using Ingenuity Pathway Analysis 331 (IPA). Here a Right-Tailed Fisher's Exact Test was used. Results were considered 332 statistically significant if the $p$-value was less than 0.05 .

Adeno-associated viruses (AAV)

335 Vectors encoding full length human WT cyclin F or cyclin F carrying the S621G mutation

336 (n-terminal V5-tagged) was cloned into a rAAV vector under the human synapsin 337 promoter using the plasmid pAAV-hSyn-EGFP (gift from Bryan Roth; Addgene, \#50465) 338 as backbone and removing EGFP. The same vector with EGFP expression was used as 339 control. Packaging of rAAV9 vectors were performed as previously described (37) using 340 the capsid AAV9.PHP.B (38). Briefly, HEK293T cells in $15 \mathrm{~cm}$ dishes were each 341 transfected with $12.5 \mu \mathrm{g}$ of vector plasmid containing gene of interest, $25 \mu \mathrm{g}$ of $\mathrm{pF} \Delta 6$ and $34212.5 \mu \mathrm{g}$ of AAV rep-cap using PEI-max in IMDM (Sigma-Aldrich). Cells were harvested 48 343 hours post-transfection by scraping and centrifuged at $350 \times \mathrm{g}$ for 30 minutes. The cell 344 supernatant was subjected to polyethylene glycol (PEG) precipitation and cell pellet was 345 further lysed using a freeze-thaw cycle and combined with the PEG mixture. After lysis 346 with sodium deoxycholate and 3 rounds of freeze/thaw cycles, the supernatant was 347 collected for purification in an OptiSeal tube (Beckman-Coulter) containing iodixanol 348 layers (15\%, 25\%, 40\%, 54\%; Sigma-Aldrich). Purified virus was collected using a 19G 349 syringe, inserted just below the 405 gradient and during dialyzed and concentrated using 
350 Amicon Ultra-15 CFU with $100 \mathrm{kDa}$ cutoff filter (Millipore). The virus was sterile filtered

351 through a Spin-X $0.22 \mu \mathrm{m}$ centrifuge filter (Corning).

352

353 AAV transduction into primary neurons

354 For AAV transduction, cortical neurons were transduced with WT CCNF, CCNF S621G or

355 EGFP AAV at MOI of 5000 on DIV 3. At 10 days in vitro (DIV), cells were treated with 0.2

$356 \mu \mathrm{M}$ of proteasome inhibitor, MG132, for 12 hours.

357

358 Immunohistochemistry and microscopy

359 Post-mortem paraffin-embedded cervical spinal cord sections from ALS patients $(n=4)$ and

360 controls $(n=3)$ were obtained from the New South Wales Brain Bank Network. For

361 immunohistochemical staining, tissue sections were heated at $70^{\circ} \mathrm{C}$ for 30 minutes,

362 deparaffinized with xylene and rehydrated with a descending series of ethanol washes. To

363 retrieve antigens, sections were boiled for 20 minutes in low $\mathrm{pH}$ buffer (pH 6.1; Dako, CA,

364 USA). Endogenous peroxidase activity and non-specific binding were blocked by

365 incubation with $3 \%$ hydrogen peroxide in methanol for 15 minutes followed by $5 \%$ normal

366 goat serum (Vector Laboratories, CA, USA) with $0.1 \%$ TWEEN-20 in PBS for 1 hour.

367 Sections were incubated at $4^{\circ} \mathrm{C}$ overnight with primary antibody rabbit anti-RBM14 (1:100,

368 Sigma-Aldrich) and then at room temperature for 1 hour with biotinylated goat anti-rabbit

369 IgG (Vector Laboratories). The avidin-biotin complex detection system (Vector

370 Laboratories) with 3,3'-diaminobenzide as chromogen (Dako) was used to detect the

371 immunoreactive signal. Nuclei were counterstained with hematoxylin before sections were

372 dehydrated with an increasing series of ethanol washes followed by xylene. Sections were

373 coverslipped using Di-N-Butyle Phthalate in xylene (DPX, Dako). 
375 Tissue sections were visualized using the ZEISS Axio Imager 2 microscope and analysed

376 using Fiji Image J. To quantify RBM14 neuronal nuclei staining, each image was first

377 deconvoluted with the Image J 'H DAB' Deconvolution Macro (39). A region of interest

378 (ROI) was drawn around the neuron nucleus and pixel intensity was scored using the IHC

379 Profiler plugin categorizing overall RBM14 staining in the ROI as either high positive,

380 positive, low positive or negative (40). Only neurons with a clear nucleus were included.

381 The number of neurons with RBM14 expression in the high positive and positive zone or

382 low positive and negative zone were plotted and analysed with Fishers' exact test

383 (significance set to 0.0166667 after Bonferroni correction).

384

385

386

387

388

389

390

391

392

393

394

395

396

397

398

399 


\section{RESULTS}

401 BiolD identifies known and novel protein interaction partners of cyclin F

402 In order to identify transient and low abundance interaction partners of cyclin F, we used a

403 proximity-based biotinylation method known as BiolD (31). Here, we first cloned cyclin F in

404 frame with a modified biotin ligase (denoted Bir $A^{*}$-cyclin $\mathrm{F}^{\mathrm{WT}}$ ). In addition, we cloned a

405 mutant of cyclin $\mathrm{F}^{\mathrm{LP} / \mathrm{AA}}$ (which has previously been reported to stabilize the interaction

406 between cyclin $\mathrm{F}$ and transiently interacting proteins) in frame with Bir ${ }^{*}$, generating

407 BirA*-Cyclin F F $^{\mathrm{LPA}}$ (Figure 1a). In order to tightly control the expression of the fusion

408 protein in cultured cells, we generated stably transfected HEK293 T-Rex Flp-In cell lines

409 (Thermo). The T-Rex Flp-In system was selected as it ensures that only a single copy of

410 the transgene is placed within the exact same insertion site in the host genome, whilst the

411 expression of cyclin $\mathrm{F}$, a cell cycle regulator, is controlled in a tetracycline-dependent

412 manner.

413

414 To conduct the BiolD experiments, transgene expression was firstly induced in HEK293

415 Flp-In T-Rex cells using tetracycline. This is followed by addition of biotin to cell culture

416 media for 24 hours. The resulting cells were lysed in harsh lysis buffer, before the

417 biotinylated proteins were isolated using streptavidin-coated beads. These resulting

418 proteins are then analysed by immunoblotting and mass spectrometry (Figure 1B). To

419 begin BiolD experiments that identify binding partners of cyclin F, we first confirmed that

420 there was no leakage in either transgene expression or biotin-labelling, whilst initiation of

421 cyclin F-BirA* expression and the addition of biotin leads to the biotinylation of proteins

422 (Figure 1C). To identify proteins in proximity to cyclin F, cells expressing either cyclin F-

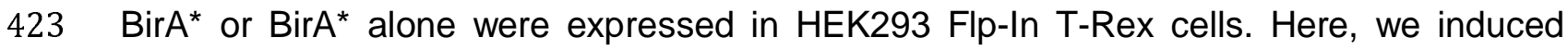

424 expression of the transgene with tetracycline, added biotin, and after 24 hours cells were 
425 harvested. Biotinylated proteins were enriched using Streptavidin Magnetic Beads

426 (Thermo) and prepared for immunoblotting and subsequent proteomic analysis. Notably,

427 immunoblotting revealed that the biotinylation profile was greater in BirA* only expressing

428 cells (Figure 1D), which correlated with higher expression levels of BirA* compared to

429 BirA*-cyclin F. Thus, prior to MS analysis we adjusted the input of isolated biotinylated

430 proteins accordingly (Supplementary Figure 1).

431

432 We carried out an in-gel trypsin digestion of the biotinylated proteins followed by liquid 433 chromatography-mass spectrometry (LC-MS/MS). In total, 918 proteins were identified, 434 with 163 proteins found in cyclin F-BirA* and cyclin F-BirA* (LP/AA) combined, but not 435 when biotin ligase was expressed alone (Figure 2A). The list of protein identifications was 436 further filtered such that proteins were considered interaction partners of cyclin $\mathrm{F}$ if they: $i$. 437 increased at least two-fold when comparing Bir ${ }^{*}$-cyclin $\mathrm{F}$ to BirA* and ii. were present in 438 at least 2 out of 3 biological replicates of BirA*-cyclin F expressing cells. The final list of 439 high-confidence interactors of cyclin F yielded 119 proteins (presented in Supplementary 440 Table 1). Within this list, we identified cyclin F and RING-box protein 1 (Rbx1). Rbx1 and 441 cyclin $\mathrm{F}$ are both essential units of the Skp1-Cul1-Fbx ${ }^{\text {(cyclin F) }} \mathrm{E} 3$ ubiquitin ligase complex, 442 confirming that the BiolD assay has biotinylated proteins within close proximity as 443 expected. Casein kinase II (CKII), another previously identified binding-partner of cyclin F $444(26,41)$, was also found in this list. 
450 Bioinformatic pathway analysis identifies known and novel functions of cyclin F

451 Next, Ingenuity Pathway Analysis (IPA) analysis was used to assign statistically significant

452 molecular functions to proteins associated with cyclin F (Figure 2B). Consistent with

453 known function of cyclin F, IPA unveiled a statistical enrichment of interaction partners

454 with roles in 'Cell Cycle Progression' ( $p<3.51 E-04)$ (Figure $2 \mathrm{C}$ ), as well as 'DNA

455 Replication, Recombination and Repair' ( $\mathrm{p}<1.59 \mathrm{E}-02)$. Several novel molecular functions

456 were also identified, one of which involves 'RNA Post-Transcriptional Modification' with

457 specific functions of 'Processing of mRNA' $(p=4.92 E-07)$, 'Splicing of mRNA' $(p=1.41 E$ -

458 06), 'Processing of $r R N A$ ' $(p=1.82 E-04)$, 'Unwinding of mRNA' ( $p=1.56 E-02)$, 'Annealing of

459 hnRNA' ( $p=1.56 E-02)$, 'Processing of RNA' $(p=1.90 E-09)$ and 'Splicing of RNA' $(p=2.70 E-$

460 07).

461

462 We also used IPA to analyse the 111 proteins that were uniquely associated with cyclin

$463 \mathrm{~F}^{\mathrm{LP} / \mathrm{AA}}$, as this protein list includes stabilized interaction partners. In this list, we found a

464 series of proteins which had roles in 'RNA Damage and Repair' ( $p=4.27 E-10)$, 'Processing

465 of RNA' ( $p=1.38 \mathrm{E}-08)$, 'Processing of $r$ RNA' $(p=7.98 \mathrm{E}-08)$, 'Splicing of RNA' (1.83E-04).

466 Within the list of proteins with roles known to be involved in RNA metabolism, there were

467 also a series of RNA binding proteins including TAF15, EWS and RBM14; proteins also

468 known to form paraspeckles (Figure 3). Notably, IPA predicted changes in the

469 homeostasis of these proteins to affect the expression of RNA, prompting further

470 investigation into the relationship between cyclin $\mathrm{F}$ and these interaction partners.

472 Cyclin F is closely associated with paraspeckle proteins

473 Given that RBM14 is essential for building subnuclear paraspeckles (42), we validate the 474 interaction between cyclin $\mathrm{F}$ and RBM14 using standard immunoprecipitation and 
475 immunoblotting (Figure 4A). Here we noted that cyclin F(WT)-flag and cyclin F(S621G)-

476 flag could co-immunoprecipitate with endogenous RBM14, with no effect from the

477 mutation. In addition, both overexpressed cyclin F(WT)-flag and cyclin F(S621G)-flag

478 could co-immunoprecipitate with RBM14-HA. Conversely, RBM14-HA could co-

479 immunoprecipitate with both cyclin F(WT)-flag and cyclin F(S621G)-flag (Figure 4B).

480

481 Next we questioned whether cyclin F could also bind other essential components of the 482 paraspeckle complex, NONO and SFPQ. Indeed, mCherry-cyclin F was found to 483 immunoprecipitate with these essential paraspeckle components in addition to RBM14 484 (Figure 4C). In all cases, both cyclin $\mathrm{F}^{\mathrm{WT}}$ and cyclin $\mathrm{F}^{\mathrm{S} 621 \mathrm{G}}$ were able to co485 immunoprecipitate with these paraspeckle components. Together the data validated the 486 mass spectrometry data and further demonstrated that cyclin F interacted with protein 487 components of the paraspeckle complex.

489 Cyclin $F^{S 621 G}$ causes disruption of paraspeckle homeostasis in primary neurons

490 Previously it has been established that proteasome inhibitor, MG132 is able to initiate 491 paraspeckle assembly and lead to elongation of the paraspeckle structure. During this 492 time, paraspeckle protein levels remain largely consistent (43). To assess the effect of 493 cyclin $\mathrm{F}^{\mathrm{S} 621 \mathrm{G}}$ on paraspeckle regulation in response to MG132, we overexpressed cyclin $494 \mathrm{~F}^{\mathrm{WT}}$, cyclin $\mathrm{F}^{\mathrm{S} 621 \mathrm{G}}$ or an empty vector in primary mouse cortical neurons by AAV infection, 495 then collected protein lysates following treatment with $0.2 \mu \mathrm{M}$ of MG132 or a vehicle 496 control (Figure 5A). Notably we observed that, in response to MG132 treatment, there was 497 a significant increase in RBM14 of 1.54 fold (Figure 5B) as well as a significant increase in 498 SFPQ levels of 1.21 fold-change (Figure $5 \mathrm{C}$ ) in cyclin $\mathrm{F}^{\mathrm{S} 621 \mathrm{G}}$ overexpressing cells 499 compared to the wild-type control. There was no significant difference in the expression of 
500 NONO in response to MG132 treatment (Figure 5D) suggesting that mutant cyclin F may

501 lead to a disruption in the homeostasis of some essential paraspeckle components.

502

503 RBM14 homeostasis is dysregulated in the motor cortex and spinal cord of ALS patients

504 RBM14 homeostasis has not previously been reported in patient postmortem tissue. To

505 determine whether RBM14 levels are dysregulated in post-mortem ALS patient tissue, we

506 measured RBM14 levels in motor cortex and spinal cord neurons via semi-quantification

507 of immunohistochemical labeling. Since RBM14 is a known nuclear protein, we

508 specifically compared RBM14 expression in neuronal nuclei from control and ALS patients

509 (Table 2). In control spinal cord neurons, RBM14 showed either primarily nuclear staining

510 or staining in both nuclei and cytoplasm. In comparison, in ALS patient tissues, there was

511 a significant reduction of nuclear RBM14 in spinal cord neurons (Figure 6).

\section{DISCUSSION}

514 In this study, we have identified novel protein interactors of cyclin F using BiolD coupled 515 with mass spectrometry. We found that cyclin F was closely associated with paraspeckle

516 proteins including RBM14, NONO, and SFPQ. Furthermore, we demonstrate that the

517 homeostasis of RBM14 and SFPQ, essential components of the paraspeckle complex, is

518 influenced by cyclin $\mathrm{F}$ and becomes defective when cyclin $\mathrm{F}$ carries an S621G mutation

519 linked to ALS/FTD. Finally, we show yet another paraspeckle protein, RBM14, may be

520 involved in ALS pathogenesis through the dysregulation of protein levels in post-mortem

521 motor cortex and spinal cord of ALS patients.

523 We previously reported the identification of disease-causing variants in CCNF in familial

524 and sporadic ALS/FTD patients and have reported defects in major protein degradation 
525 systems in cells overexpressing cyclin F(S621G) (1). Given these known deficits, it is

526 logical to predict that the role of cyclin F in ALS pathogenesis may be associated with

527 defective protein degradation pathways. Given that many interaction partners of cyclin $\mathrm{F}$

528 were unknown, we undertook unbiased proteomic screening to identify interaction

529 partners cyclin F. To take advantage of the T-Rex and Flp-In systems (allowing controlled

530 copy number integration at the exact same site) we performed the BiolD assay in HEK293

531 cells, and in doing so, identified more than 100 high-confidence interaction partners. We

532 acknowledge that many of these may not be relevant to motor neurons as their biological

533 function is related to activities such as cell division. However, we did identify several

534 proteins involved in RNA processing pathways that are likely to be relevant to ALS/FTD.

535

536 The BiolD assay identified a close association of cyclin $\mathrm{F}$ with a group of paraspeckle

537 proteins. Further work revealed that an ALS-causing mutation in cyclin $F$ leads to the

538 defective homeostasis of essential paraspeckle proteins, RBM14 and SFPQ.

539 Paraspeckles are a class of subnuclear bodies that form within the interchromatin space

540 of mammalian cells (44). These RNA-protein structures form as RNA binding proteins

541 interact with the long non-coding RNA (IncRNA), NEAT1 (45). Alterations to paraspeckle

542 assembly and function has important implications in the context of neurodegeneration as

543 paraspeckles have a clear role in controlling gene expression (44). In particular,

544 paraspeckles are known to regulate multiple cellular processes such as cell stress

545 responses, cellular differentiation and viral infections (44). Therefore, disruption to

546 paraspeckle assembly or function results in inability to rapidly transcribe stress-responsive

547 proteins required for maintaining cellular viability. Notably, the formation of paraspeckles,

548 and the dysregulation of this process, is emerging as a biological marker of ALS. For

549 example, the assembly of paraspeckle proteins around NEAT1_2 has been reported in

550 spinal motor neurons of early-stage ALS patients (46). In addition, compromised 
551 paraspeckle formation has been identified in cell and animal models of FUSopathies, with

552 mislocalised FUS resulting in neuronal inclusions of paraspeckle components (47). In both

553 studies, the increased levels of paraspeckle assembly may represent a downstream,

554 protective cellular response to stress. We now report a different type of possible

555 involvement of paraspeckles in ALS pathogenesis. We show that RBM14 homeostasis is

556 dysregulated in post-mortem brain and spinal cord of ALS patients. RBM14 has been

557 shown to connect key paraspeckle subcomplexes, a function which requires the presence

558 of its prion-like domain (42). Thus, the dysregulation of RBM14 (and potentially other

559 paraspeckle proteins), a core paraspeckle protein, may impair paraspeckle

560 assembly/function and leave motor neurons vulnerable to cellular stress and therefore

561 more susceptible to neurodegeneration. Importantly, in this study, we have shown that

562 RBM14 dysregulation occurs in the brain and spinal cord of patients, regardless of the

563 presence of CCNF mutation, suggesting that the dysregulation of RBM14 homeostasis

564 may be one contributing step in the multi-stage pathogenesis of ALS.

565 Of the more than 30 genes (and their corresponding protein products) that are now linked

566 to ALS, two broad functional categories have emerged; protein-degradation pathways

567 (indirectly because of the presence of abnormal protein aggregates, and directly through

568 regulators of protein degradation such as cyclin $\mathrm{F}$ and ubiquilin-2) and RNA processing.

569 However, the link between these two distinct groups of proteins remain poorly understood.

570 Perhaps the strongest association to date is represented by TDP-43 and to a lesser extent

571 FUS, which are both major constituents of intraneuronal aggregates, and their core

572 function being associated with RNA processing (48). We now present a new and different

573 linkage, demonstrating that cyclin $\mathrm{F}$ influences the homeostasis of key paraspeckle

574 components. Future studies should look to identify the cellular processes, such as RNA

575 processing in response to stress stimuli, that may become dysregulated due to the 
576 reduction of RBM14 in the nucleus of affected motor neurons, as this may provide deeper

577 insight into the underlying causes of ALS/FTD. Notably, this study also identifies RBM14

578 dysregulation in sporadic cases of ALS. Together the data further link the dysregulation of

579 the ubiquitin-proteasome system and RNA processing to the pathogenesis of ALS/FTD.

580

581 CONCLUSIONS

582 This study employed an unbiased proteomic screening assay which revealed that cyclin $\mathrm{F}$

583 interacts with several core proteins of the paraspeckle complex. Using

584 immunoprecipitation, we confirmed the interaction between cyclin $\mathrm{F}$ and three paraspeckle

585 proteins; RBM14, NONO and SFPQ. Notably, we demonstrate that the pathogenic cyclin

$586 \mathrm{~F}^{\mathrm{S} 621 \mathrm{G}}$ variant disrupts the homeostasis of these proteins and their responsiveness to a

587 stressor that stimulates paraspeckle formation. Finally, we report for the first time that

588 RBM14 levels are dysregulated in brain and spinal cord of ALS patients relative to healthy

589 patient controls. Collectively, these data suggest that cyclin F may influence stress

590 responses through modulation of the paraspeckle complex, and that disruption in

591 paraspeckle homeostasis may contribute to the molecular pathogenesis of ALS/FTD. 


\begin{tabular}{lll}
598 & Abbreviations \\
599 & AAV & Adeno-associated viruses \\
600 & ACN & Acetonitrile \\
601 & AGC & Automatic gain control \\
602 & ALS & Amyotrophic Lateral Sclerosis \\
603 & AmBic & Ammonium bicarbonate \\
604 & BCA & Bicinchoninic acid assay \\
605 & BiolD & Proximity dependent Biotin ldentification \\
606 & Bis-Tris & 1,3-bis(tris(hydroxymethyl)methylamino)propane \\
607 & BSA & Bovine serum albumin \\
608 & DIV & Days in vitro \\
609 & DMEM & Dulbecco's Modified Eagle Medium \\
610 & DMSO & Dimethyl sulfoxide \\
611 & DNA & Deoxyribonucleic acid \\
612 & DTT & Dithiothreitol \\
613 & EDTA & Ethylenediaminetetraacetic acid \\
614 & EGFP & Enhanced green fluorescent protein \\
615 & FA & Formic acid \\
616 & FT-MS & Fourier transform-mass spectrometry \\
617 & FBS & Fetal bovine serum \\
618 & FDR & False discovery rate \\
619 & FTD & Frontotemporal Dementia \\
620 & HCD & Higher energy collision dissociation \\
621 & MS & Mass spectrometry \\
622 & IAA & lodoacetamide \\
\hline
\end{tabular}




$\begin{array}{lll}623 & \text { IgG } & \text { Immunoglobulin G } \\ 624 & \text { IMDM } & \text { Iscove's Modified Dulbecco's Medium } \\ 625 & \text { IP } & \text { Immunoprecipitation } \\ 626 & \text { IPA } & \text { Ingenuity Pathway Analysis } \\ 627 & \text { LFQ } & \text { Label-free quantitation } \\ 628 & \text { MND } & \text { Motor neurone disease } \\ 629 & \text { MOI } & \text { Multiplicity of Infection } \\ 630 & \text { nanoESI } & \text { Nanoelectrospray ionization } \\ 631 & \text { NHEJ } & \text { Non-Homologous End Joining } \\ 632 & \text { NP-40 } & \text { Nonidet P-40 } \\ 633 & \text { PBS } & \text { Phosphate buffered saline } \\ 634 & \text { PBST } & \text { Phosphate buffered saline containing Tween-20 } \\ 635 & \text { PEG } & \text { Polyethylene glycol } \\ 636 & \text { PEI } & \text { Polyethylenimine } \\ 637 & \text { PSM } & \text { Peptide-spectrum match } \\ 638 & \text { RIPA } & \text { Radioimmunoprecipitation assay buffer } \\ 639 & \text { ROI } & \text { Region of interest } \\ 640 & \text { SCF } & \text { Skp1-Cul1-F-Box } \\ 641 & \text { SDS } & \text { Sodium dodecyl sulphate } \\ 642 & \text { SDS-PAGE } & \text { Sodium dodecyl sulphate polyacrylamide gel electrophoresis } \\ 643 & \text { UPS } & \text { Ubiquitin-proteasome system } \\ 6\end{array}$

644

645

646

647 


\section{DECLARATIONS}

649 Ethics approval and consent to participate

650 International, national, and/or institutional guidelines for the care and use of animals were

651 followed. Ethics approval was also obtained for the use of human tissue.

652 Consent for publication

653 Not applicable

654 Availability of data and material

655 The mass spectrometry proteomics data have been deposited to the ProteomeXchange

656 Consortium via the PRIDE partner repository with the dataset identifier PXD014163 and

657 10.6019/PXD014163.

658 Competing interests

659 The authors declare that they have no competing interests.

$660 \quad$ Funding

661 This research has been supported by research grants from the National Health \& Medical

662 Research Council (APP1095215, APP1107644), Motor Neurone Disease Research

663 Institute of Australia (GIA1510, GIA1628, GIA1715 and IG1910), and philanthropic

664 donations to the Macquarie University Centre for MND Research.

\section{Authors' Contributions}

666 R.C. and A.L. and S.L.R. conceptualized the project. S.L.R. conducted the BiolD studies, 667 MS analysis, follow-up biochemical studies and wrote the manuscript. F.C. assisted with 668 MS sample runs. A.D. assisted with stable cell line generation. S.Y. and N.G. generated 669 Iysates from patient tissue and conducted IHC studies using patient tissue. C.G.A. and 670 Y.D.K. conducted primary neuron transduction and drug treatment. M.P.M., I.B., A.L., 
bioRxiv preprint doi: https://doi.org/10.1101/2020.04.30.071365; this version posted May 1, 2020. The copyright holder for this preprint (which

was not certified by peer review) is the author/funder, who has granted bioRxiv a license to display the preprint in perpetuity. It is made available under aCC-BY-NC-ND 4.0 International license.

671 R.C., J.M.D., M.M., L.M.I., B.S. assisted with editing the manuscript. All authors read and

672 approved the final manuscript.

673

674

675 Acknowledgements

676 This research was supported by access to the Australian Proteomics Analysis Facility

677 (APAF) established under the Australian Government's NCRIS program.

678

\section{REFERENCES}

680 1. K. L. Williams et al., CCNF mutations in amyotrophic lateral sclerosis and

681 frontotemporal dementia. Nature communications 7, 11253 (Apr 15, 2016).

682 2. A. Lee et al., Pathogenic mutation in the ALS/FTD gene, CCNF, causes elevated

683 Lys48-linked ubiquitylation and defective autophagy. Cellular and molecular life

684 sciences: CMLS, (Aug 29, 2017).

685 3. R. Ferrari, D. Kapogiannis, E. D. Huey, P. Momeni, FTD and ALS: a tale of two

686 diseases. Current Alzheimer research 8, 273 (May, 2011).

687 4. D. R. Rosen et al., Mutations in $\mathrm{Cu} / \mathrm{Zn}$ superoxide dismutase gene are associated

688 with familial amyotrophic lateral sclerosis. Nature 362, 59 (Mar 4, 1993).

689 5. H. X. Deng et al, Amyotrophic lateral sclerosis and structural defects in $\mathrm{Cu}, \mathrm{Zn}$

$690 \quad$ superoxide dismutase. Science 261, 1047 (Aug 20, 1993).

691 6. G. D. Watts et al., Inclusion body myopathy associated with Paget disease of bone

692

693

694

695

696

697

698

699

700

701

702

703

704

705

706

707

708

709 and frontotemporal dementia is caused by mutant valosin-containing protein. Nature genetics 36, 377 (Apr, 2004).

7. J. Sreedharan et al, TDP-43 mutations in familial and sporadic amyotrophic lateral sclerosis. Science 319, 1668 (Mar 21, 2008).

8. T. J. Kwiatkowski, Jr. et al., Mutations in the FUS/TLS gene on chromosome 16 cause familial amyotrophic lateral sclerosis. Science 323, 1205 (Feb 27, 2009).

9. C. Vance et al., Mutations in FUS, an RNA processing protein, cause familial amyotrophic lateral sclerosis type 6. Science 323, 1208 (Feb 27, 2009).

10. H. Maruyama et al., Mutations of optineurin in amyotrophic lateral sclerosis. Nature 465, 223 (May 13, 2010).

11. F. Fecto et al., SQSTM1 mutations in familial and sporadic amyotrophic lateral sclerosis. Archives of neurology 68, 1440 (Nov, 2011).

12. H. X. Deng et al., Mutations in UBQLN2 cause dominant X-linked juvenile and adult-onset ALS and ALS/dementia. Nature 477, 211 (Aug 21, 2011).

13. J. 0. Johnson et al., Mutations in the Matrin 3 gene cause familial amyotrophic lateral sclerosis. Nature neuroscience 17, 664 (May, 2014).

14. E. T. Cirulli et al, Exome sequencing in amyotrophic lateral sclerosis identifies risk genes and pathways. Science 347, 1436 (Mar 27, 2015). 
710

711

712

713

714

715

716

717

718

719

720

721

722

723

724

725

726

727

728

729

730

731

732

733

734

735

736

737

738

739

740

741

742

743

744

745

746

747

748

749

750

751

752

753

754

755

756
15. A. Freischmidt et al., Haploinsufficiency of TBK1 causes familial ALS and frontotemporal dementia. Nature neuroscience 18, 631 (May, 2015).

16. J. Galper et al., Cyclin F: A component of an E3 ubiquitin ligase complex with roles in neurodegeneration and cancer. The international journal of biochemistry \& cell biology 89, 216 (Aug, 2017).

17. A. L. Hogan et al., Expression of ALS/FTD-linked mutant CCNF in zebrafish leads to increased cell death in the spinal cord and an aberrant motor phenotype. Human molecular genetics 26, 2616 (Jul 15, 2017).

18. V. D'Angiolella et al., Cyclin F-mediated degradation of ribonucleotide reductase M2 controls genome integrity and DNA repair. Cell 149, 1023 (May 25, 2012).

19. M. J. Emanuele et al., Global identification of modular cullin-RING ligase substrates. Cell 147, 459 (Oct 14, 2011).

20. V. D'Angiolella et al, SCF(Cyclin F) controls centrosome homeostasis and mitotic fidelity through CP110 degradation. Nature 466, 138 (Jul 1, 2010).

21. D. Walter et al, SCF(Cyclin F)-dependent degradation of CDC6 suppresses DNA re-replication. Nature communications 7, 10530 (Jan 28, 2016).

22. J. F. Dankert et al, Cyclin F-Mediated Degradation of SLBP Limits H2A.X Accumulation and Apoptosis upon Genotoxic Stress in G2. Molecular cell 64, 507 (Nov 3, 2016).

23. A. E. Elia et al., Quantitative Proteomic Atlas of Ubiquitination and Acetylation in the DNA Damage Response. Molecular cell 59, 867 (Sep 3, 2015).

24. R. Choudhury et al, APC/C and SCF(cyclin F) Constitute a Reciprocal Feedback Circuit Controlling S-Phase Entry. Cell reports 16, 3359 (Sep 20, 2016).

25. D. K. Klein et al., Cyclin F suppresses B-Myb activity to promote cell cycle checkpoint control. Nature communications 6, 5800 (Jan 5, 2015).

26. A. Lee et al., Casein kinase II phosphorylation of cyclin $\mathrm{F}$ at serine 621 regulates the Lys48-ubiquitylation E3 ligase activity of the SCF((cyclin F)) complex. Open biology 7, (Oct, 2017).

27. D. I. Kim et al., Probing nuclear pore complex architecture with proximitydependent biotinylation. Proceedings of the National Academy of Sciences of the United States of America 111, E2453 (Jun 17, 2014).

28. K. J. Roux, D. I. Kim, B. Burke, BioID: a screen for protein-protein interactions. Current protocols in protein science 74, Unit 1923 (Nov 5, 2013).

29. K. J. Roux, D. I. Kim, B. Burke, D. G. May, BioID: A Screen for Protein-Protein Interactions. Current protocols in protein science 91, 19231 (Feb 21, 2018).

30. S. Rayner et al., Using proteomics to identify ubiquitin ligase-substrate pairs: how novel methods may unveil therapeutic targets for neurodegenerative diseases. Cell Mol Life Sci. 76, 2499 (2019).

31. K. J. Roux, D. I. Kim, M. Raida, B. Burke, A promiscuous biotin ligase fusion protein identifies proximal and interacting proteins in mammalian cells. The Journal of cell biology 196, 801 (Mar 19, 2012).

32. C. M. Van Itallie et al., Biotin ligase tagging identifies proteins proximal to Ecadherin, including lipoma preferred partner, a regulator of epithelial cell-cell and cell-substrate adhesion. Journal of cell science 127, 885 (Feb 15, 2014).

33. C. M. Van Itallie et al, The $\mathrm{N}$ and $\mathrm{C}$ termini of ZO-1 are surrounded by distinct proteins and functional protein networks. The Journal of biological chemistry 288, 13775 (May 10, 2013). 
766

767

768

769

770

771

772

773

774

775

776

777

778

779

780

781

782

783

784

785

786

787

788

789

790

791

792

793

794

795

796

797

798

799

34. C. C. Chou et al., TDP-43 pathology disrupts nuclear pore complexes and nucleocytoplasmic transport in ALS/FTD. Nature neuroscience 21, 228 (Feb, 2018).

35. E. Coyaud et al., BioID-based Identification of Skp Cullin F-box (SCF) betaTrCP1/2 E3 Ligase Substrates. Molecular \& cellular proteomics : MCP 14, 1781 (Jul, 2015).

36. T. Fath, Y. D. Ke, P. Gunning, J. Gotz, L. M. Ittner, Primary support cultures of hippocampal and substantia nigra neurons. Nature protocols 4, 78 (2009).

37. M. Bi et al., Tau exacerbates excitotoxic brain damage in an animal model of stroke. Nature communications 8, 473 (Sep 7, 2017).

38. B. E. Deverman et al., Cre-dependent selection yields AAV variants for widespread gene transfer to the adult brain. Nature biotechnology 34, 204 (Feb, 2016).

39. A. C. Ruifrok, D. A. Johnston, Quantification of histochemical staining by color deconvolution. Analytical and quantitative cytology and histology 23, 291 (Aug, 2001).

40. F. Varghese, A. B. Bukhari, R. Malhotra, A. De, IHC Profiler: an open source plugin for the quantitative evaluation and automated scoring of immunohistochemistry images of human tissue samples. PloS one 9, e96801 (2014).

41. I. Mavrommati et al, beta-TrCP- and Casein Kinase II-Mediated Degradation of Cyclin F Controls Timely Mitotic Progression. Cell reports 24, 3404 (Sep 25, 2018).

42. S. Hennig et al., Prion-like domains in RNA binding proteins are essential for building subnuclear paraspeckles. The Journal of cell biology 210, 529 (Aug 17, 2015).

43. T. Hirose et al, NEAT1 long noncoding RNA regulates transcription via protein sequestration within subnuclear bodies. Molecular biology of the cell 25, 169 (Jan, 2014).

44. A. H. Fox, A. I. Lamond, Paraspeckles. Cold Spring Harbor perspectives in biology 2, a000687 (Jul, 2010).

45. C. S. Bond, A. H. Fox, Paraspeckles: nuclear bodies built on long noncoding RNA. The Journal of cell biology 186, 637 (Sep 7, 2009).

46. Y. Nishimoto et al, The long non-coding RNA nuclear-enriched abundant transcript 1_2 induces paraspeckle formation in the motor neuron during the early phase of amyotrophic lateral sclerosis. Molecular brain 6, 31 (Jul 8, 2013).

47. T. A. Shelkovnikova, H. K. Robinson, C. Troakes, N. Ninkina, V. L. Buchman, Compromised paraspeckle formation as a pathogenic factor in FUSopathies. Human molecular genetics 23, 2298 (May 1, 2014).

48. C. Lagier-Tourenne, M. Polymenidou, D. W. Cleveland, TDP-43 and FUS/TLS: emerging roles in RNA processing and neurodegeneration. Human molecular genetics 19, R46 (Apr 15, 2010). 
Table 1. Details of patient tissue

\begin{tabular}{|c|c|c|c|c|c|}
\hline Case & Tissue type & $\begin{array}{l}\text { Age } \\
(y)\end{array}$ & Gender & PMI (hr) & $\begin{array}{l}\text { Disease } \\
\text { onset }\end{array}$ \\
\hline Control & Motor cortex & 37 & Male & 24 & $\mathrm{~N} / \mathrm{A}$ \\
\hline Control & $\begin{array}{l}\text { Spinal cord and motor } \\
\text { cortex }\end{array}$ & 61 & Male & 30 & $\mathrm{~N} / \mathrm{A}$ \\
\hline Control & $\begin{array}{l}\text { Spinal cord and motor } \\
\text { cortex }\end{array}$ & 80 & Male & 12 & $\mathrm{~N} / \mathrm{A}$ \\
\hline Control & Spinal cord & 75 & Male & 34 & $\mathrm{~N} / \mathrm{A}$ \\
\hline SALS & Motor cortex & 81 & Male & 70 & 80 \\
\hline SALS & Motor cortex & 84 & Male & 23 & 74 \\
\hline FALS (C9orf72) & Motor cortex & 60 & Male & 99 & 58 \\
\hline $\begin{array}{l}\text { FALS (Unknown } \\
\text { mutation) }\end{array}$ & $\begin{array}{l}\text { Spinal cord and motor } \\
\text { cortex }\end{array}$ & 54 & Female & 16 & 53 \\
\hline SALS (C9orf72) & Spinal cord & 65 & Female & 18 & 65 \\
\hline SALS & Spinal cord & 71 & Male & 12.5 & 70 \\
\hline FALS (C9orf72) & Spinal cord & 75 & Male & 74 & 21.5 \\
\hline
\end{tabular}


bioRxiv preprint doi: https://doi.org/10.1101/2020.04.30.071365; this version posted May 1, 2020. The copyright holder for this preprint (which was not certified by peer review) is the author/funder, who has granted bioRxiv a license to display the preprint in perpetuity. It is made available under aCC-BY-NC-ND 4.0 International license. 


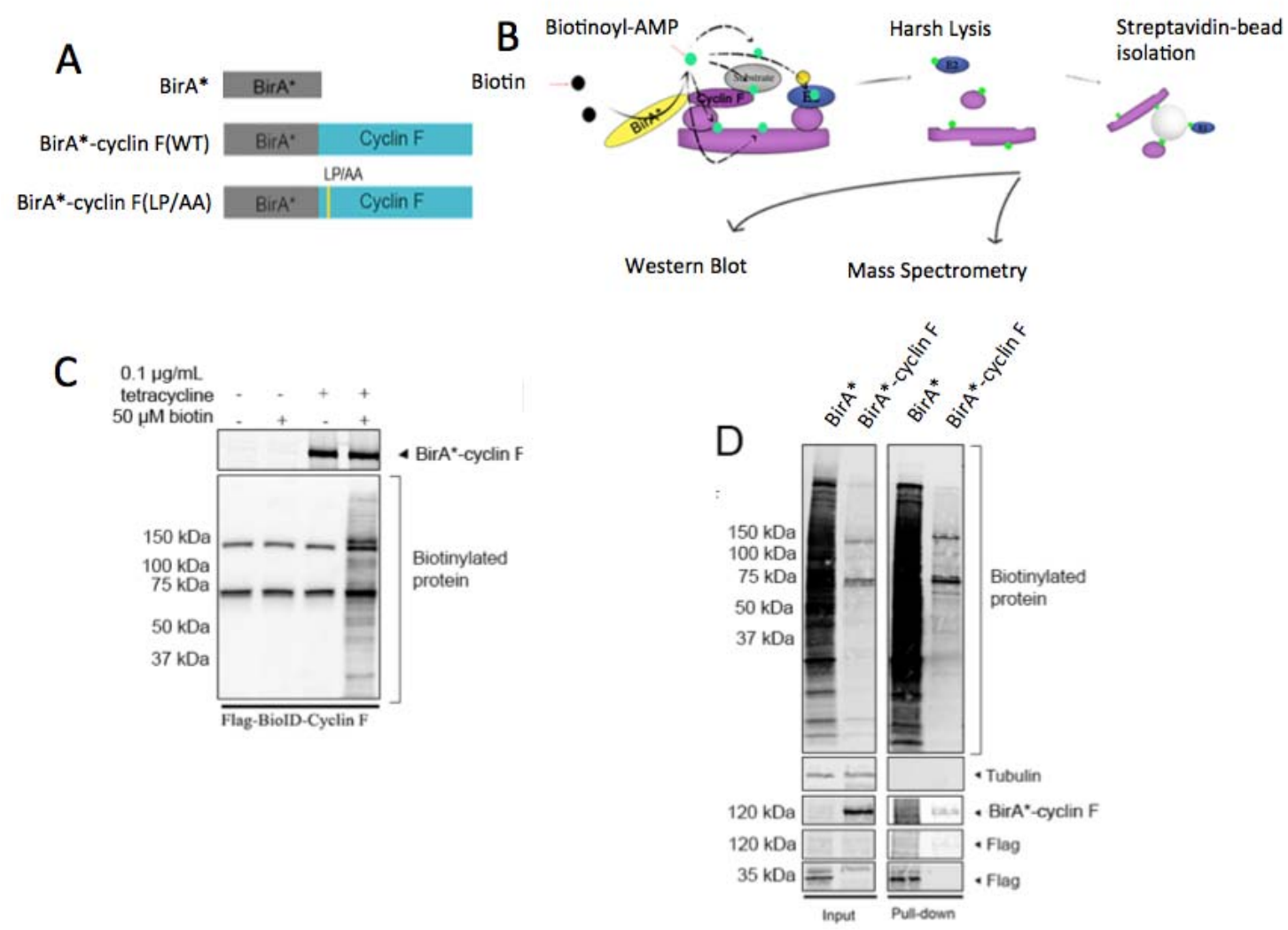

Figure 1. Identifying cyclin $F$ interaction partners using BiolD. A. BirA* alone, BirA*cyclin $\mathrm{F}(\mathrm{WT})$ and $\mathrm{Bir}^{*}$-cyclin $\mathrm{F}(\mathrm{LP} / \mathrm{AA})$ were stably transfected into Flp-In T-Rex HEK293 cells. B. Schematic showing process of biotinylation by BirA*, isolation of biotinylated proteins and identification by western blotting or mass spectrometry. C. Stably transfected HEK293 Flp-In cells were treated with tetracycline to induce gene expression. Addition of biotin led to the biotinylation of proteins in proximity to cyclin F, as detected by immunoblotting with fluorescently-tagged streptavidin (LiCor). D. The biotinylation profile of BirA* alone or cyclin F-BirA* before and after streptavidincoated bead pull-downs. 

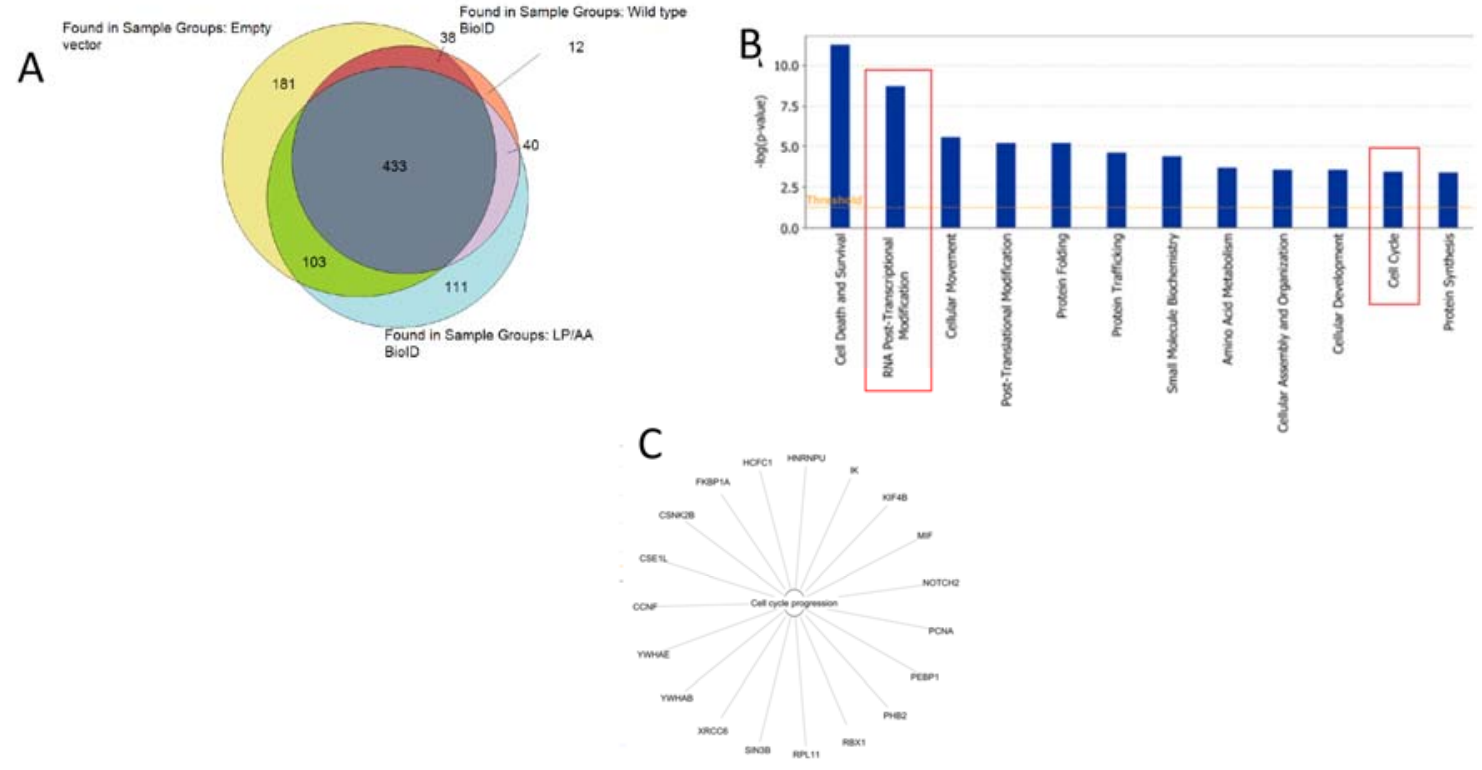

Figure 2. A. Proteomic analysis identified common and unique proteins biotinylated by BirA* alone, BirA*-cyclin $F(W T)$ and Bir $A^{*}$-cyclin F(LP/AA). B. Ingenuity Pathway analysis (IPA) of protein interaction partners showing top twelve statistically enriched molecular processes for cyclin $\mathrm{F}$ interaction partners. C. Proteins interaction partners involved in cell cycle progression. 
bioRxiv preprint doi: https://doi.org/10.1101/2020.04.30.071365; this version posted May 1, 2020. The copyright holder for this preprint (which was not certified by peer review) is the author/funder, who has granted bioRxiv a license to display the preprint in perpetuity. It is made available under aCC-BY-NC-ND 4.0 International license.
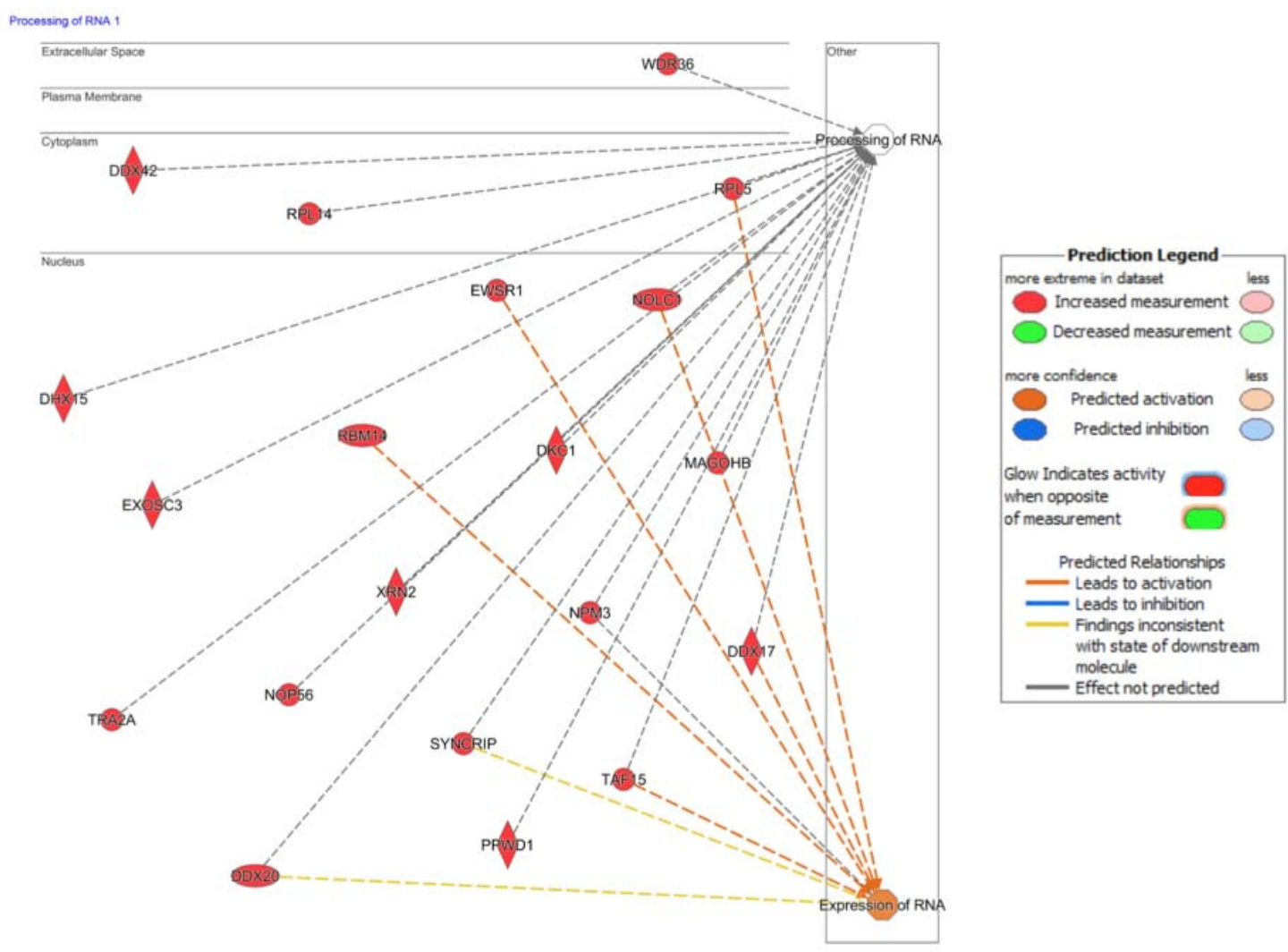

Figure 3. Protein interaction partners involved in RNA processing and expression along with subcellular localisation. 


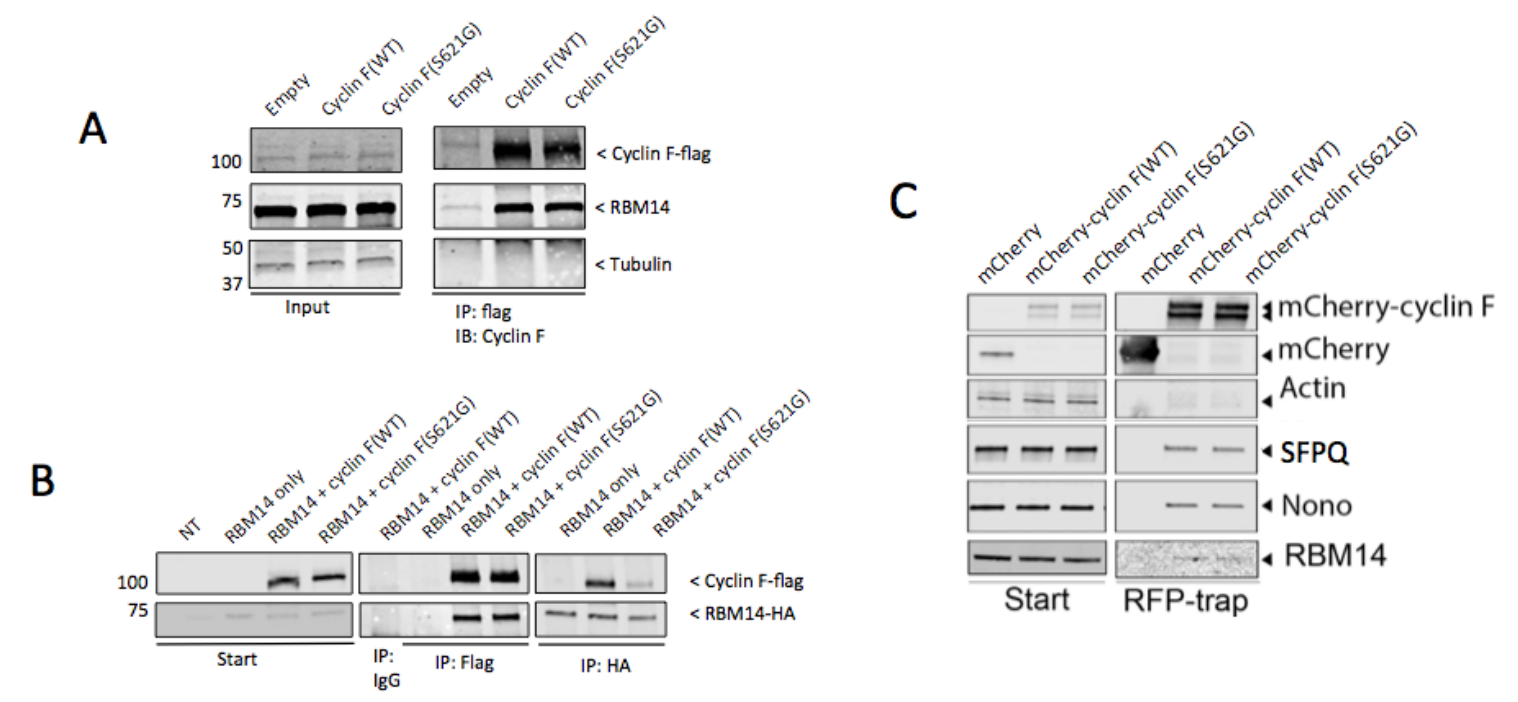

Figure 4. Cyclin F interacts with paraspeckle proteins. A. Flag-tagged cyclin F(WT), cyclin F(S621G) or an empty vector control were transfected into HEK293 cells. Anti-flag antibody was used to immunoprecipitate flag-tagged proteins. Eluates were evaluated by immunoblotting with the antibodies specified. B. Flag-tagged cyclin F(WT) or cyclin F(S621G) were co-transfected alongside RBM14-HA in HEK293 cells. Anti-flag or anti-HA antibody was used to immunoprecipitate flag-tagged or HA-tagged proteins as specified. Eluates were evaluated by immunoblotting with the antibodies specified. C. mCherry-cyclin F(WT) or mCherry-cyclin F(S621G) were transfected into HEK293 cells. An RFP-trap was incubated with lysates to immunoprecipitate mCherry-tagged proteins. Eluates were evaluated by immunoblotting using the antibodies specified. 

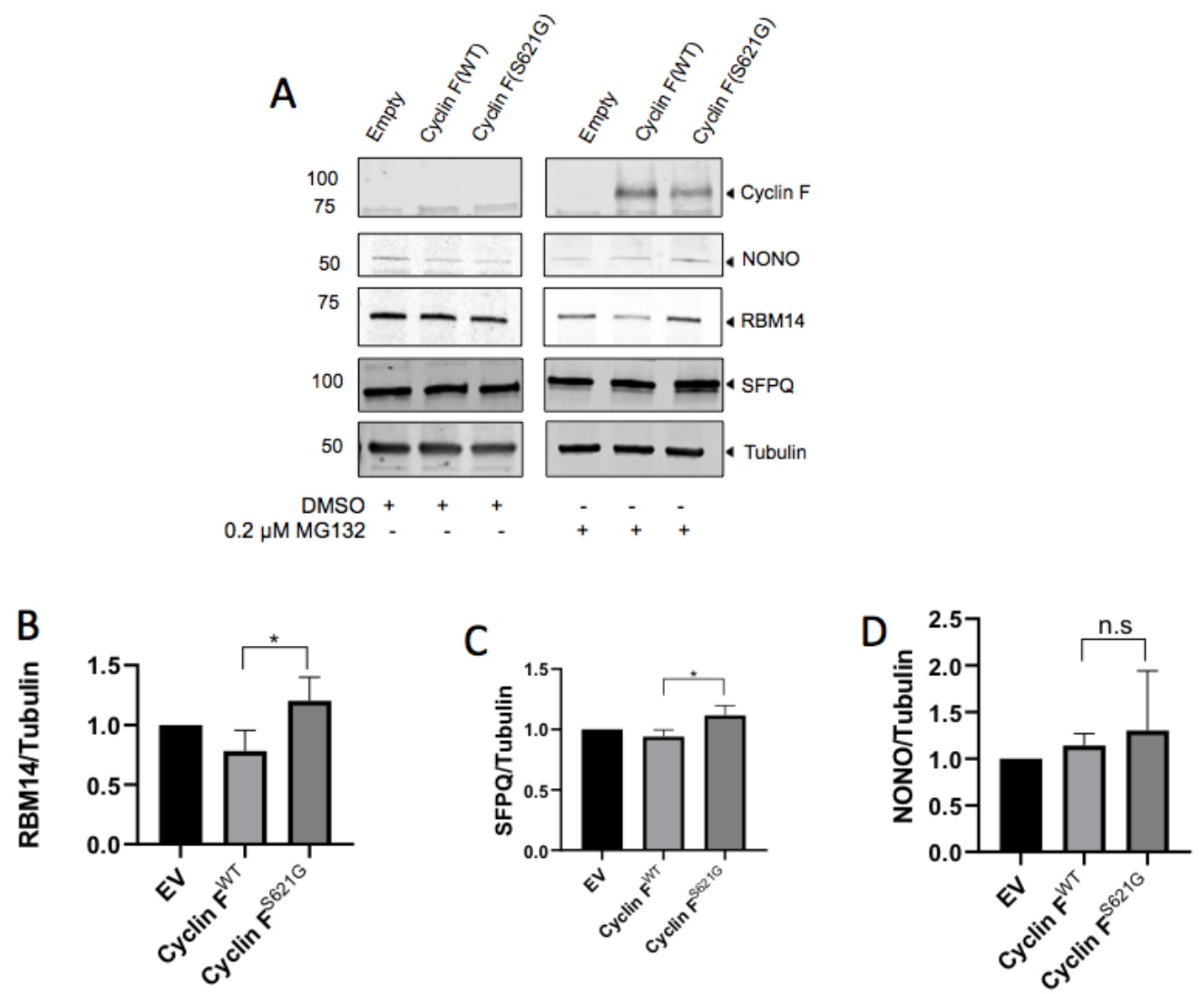

Figure 5. Cyclin $\mathrm{F}^{\mathrm{S} 621 \mathrm{G}}$ causes defective turnover of paraspeckle components in primary neurons. A. Primary neurons were transduced using constructs encoding cyclin $\mathrm{F}^{\mathrm{WT}}$, cyclin $\mathrm{F}^{\mathrm{S} 621 \mathrm{G}}$ or an empty vector control. Transduced neurons were treated with $0.2 \mu \mathrm{M}$ MG132 or a vehicle control for 24 hours before cells were lysed in RIPA buffer and analysed by immunoblotting using the antibodies as indicated. B. Densitometry of RBM14 upon MG132 treatment normalised to Tubulin $\left(n=4,{ }^{*}: p<0.05\right)$. C. Densitometry of SFPQ upon MG132 treatment normalised to Tubulin $\left(n=4,{ }^{*}: p<0.05\right)$. D. Densitometry of NONO upon MG132 treatment normalised to Tubulin ( $n=4$, n.s; not significant). 

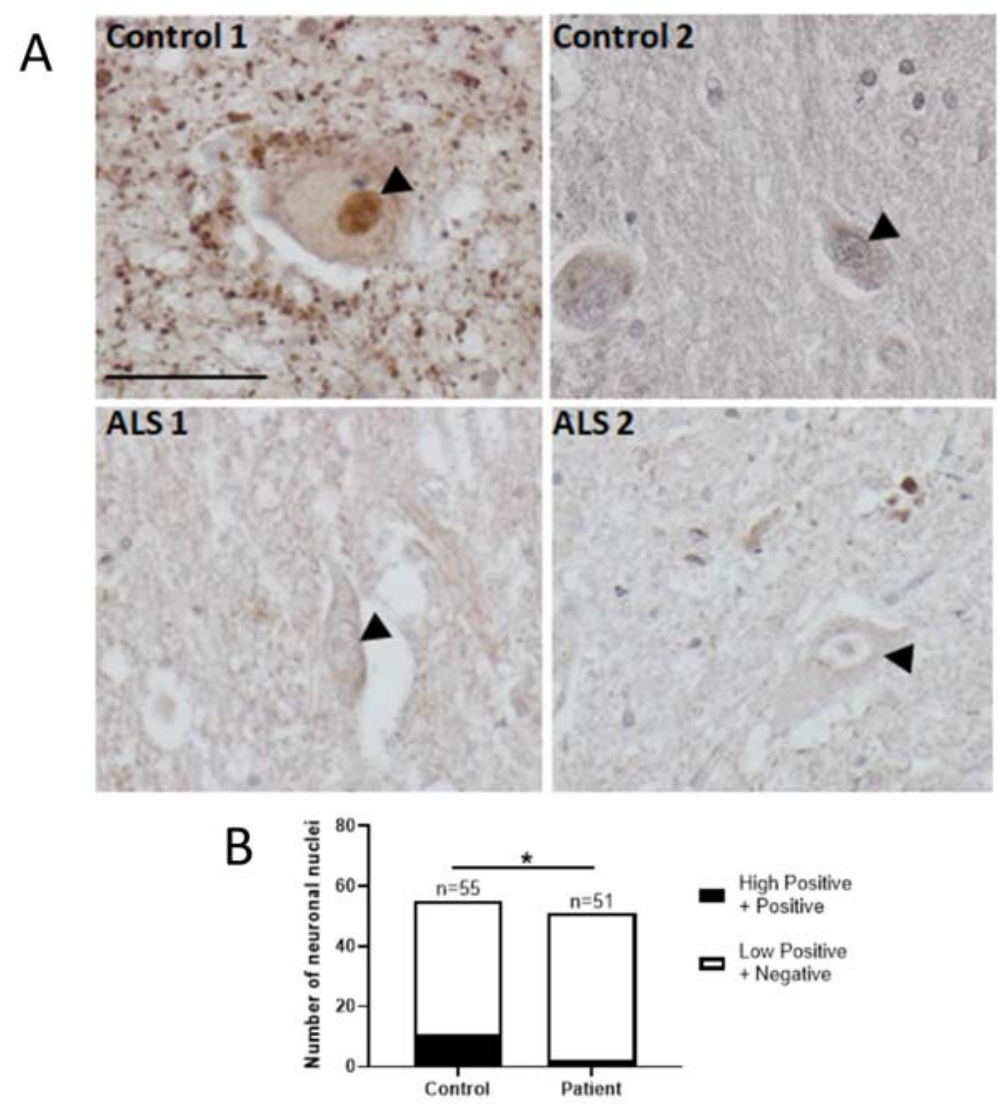

Figure 6. RBM14 is reduced in the brain and spinal cord of ALS patients. A. Representative images of RBM14 immunohistochemical staining in control $(n=3)$ and ALS patient $(n=4)$ spinal cord tissues. Arrowheads indicate RBM14 staining in neuronal nuclei. Scale bar=50 $\mu \mathrm{m}$. B. Semi-quantification of RBM14 IHC staining showed a significant reduction of RBM14 in neuronal nuclei from ALS patients compared to controls. A total number of 55 control neurons and 51 patient neurons were analysed using Image $\mathrm{J} \mathrm{IHC}$ Profiler Plugin (Fisher's exact test, * $p<0.05$ ). 